\title{
Snapping of the Proximal Hamstring Origin: A Rare Cause of Coxa Saltans
}

\author{
A Case Report \\ Anthony Scillia, MD, Andrew Choo, MD, Edward Milman, MD, Vincent McInerney, MD, and Anthony Festa, MD \\ Investigation performed at Seton Hall University School of Health and Medical Sciences, South Orange, \\ and St. Joseph's Regional Medical Center, Paterson, New Jersey
}

$\mathrm{T}$ he term "snapping bottom" was initially used by Rask' to describe subluxation of the long head of the biceps femoris tendon at the ischial tuberosity, in what we believe to be the only reported case of this phenomenon in the literature. This entity was discovered by reproduction of the snapping during active hip flexion and with direct palpation of the snapping long head of the biceps femoris tendon over the ischial tuberosity. After unsuccessful nonoperative treatment, a tenotomy was performed; all symptoms were allevi- ated. There are several etiologies of the snapping hip, "coxa saltans," which include snapping of the iliotibial band or gluteus maximus over the greater trochanter, snapping of the the iliopsoas over the iliopectineal eminence, and intra-articular lesions ${ }^{2}$. However, subluxation of the proximal hamstring origin is rarely considered in the differential diagnosis. We present the case of a woman with coxa saltans caused by subluxation of the proximal hamstring origin over the ischial tuberosity. Institutional review board approval and the

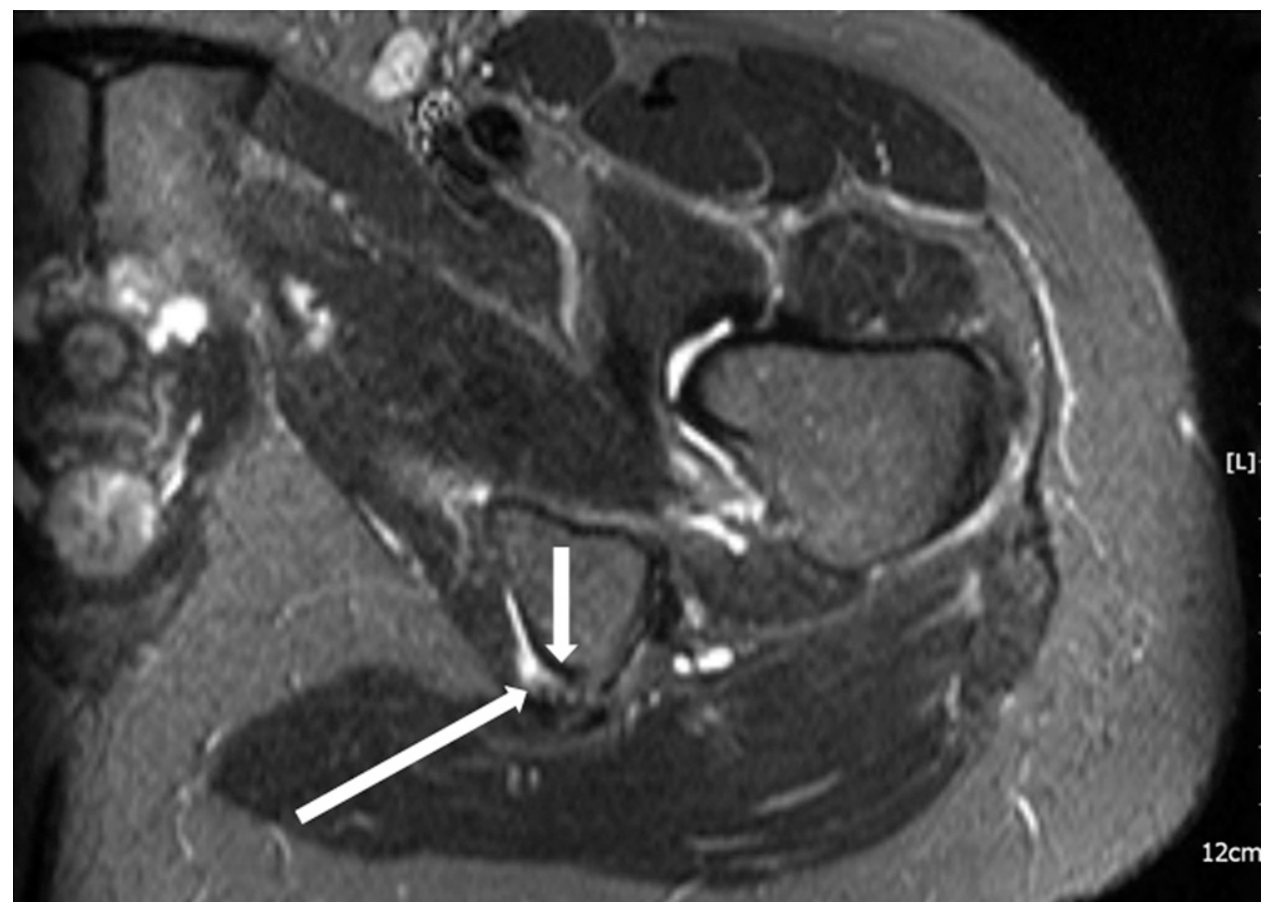

Fig. 1

Axial fluid-sensitive MRI demonstrating the partial tear of the proximal hamstring origin at the ischial tuberosity. The long arrow indicates the elongated, partially torn semimembranosus tendon, with abnormal fluid around the ischial tuberosity. The short arrow points to the posterior cortex of the ischial tuberosity.

Disclosure: None of the authors received payments or services, either directly or indirectly (i.e., via his or her institution), from a third party in support of any aspect of this work. None of the authors, or their institution(s), have had any financial relationship, in the thirty-six months prior to submission of this work, with any entity in the biomedical arena that could be perceived to influence or have the potential to influence what is written in this work. Also, no author has had any other relationships, or has engaged in any other activities, that could be perceived to influence or have the potential to influence what is written in this work. The complete Disclosures of Potential Conflicts of Interest submitted by authors are always provided with the online version of the article. 
A

B

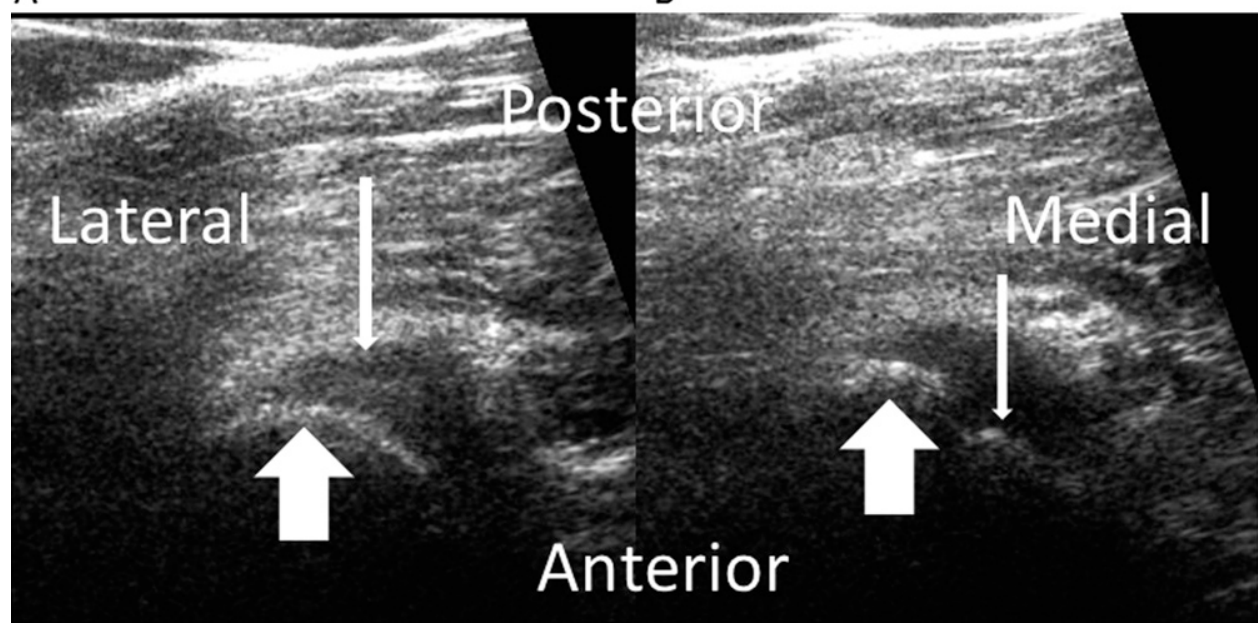

Fig. 2

Still images from a dynamic ultrasound study with the patient standing erect (Fig. 2-A) and with full hip flexion (Fig. 2-B), with the ultrasound probe over the gluteal region. The short arrow indicates the ischial tuberosity cortex at the hamstring origin. The long arrow indicates the medially subluxated, partially torn tendon.

patient's informed consent for publication of this information were obtained.

\section{Case Report}

A fifty-five-year-old female recreational tennis player presented $\mathrm{A}_{\text {to }}$ us with a one-year history of left buttock pain as well as audible snapping of the buttock when she bent at the hips. The symptoms began approximately one month after she sustained a hamstring strain while playing tennis. Because of the pain, she had been unable to return to athletic activities. Physical examination revealed tenderness at the hamstring origin. Full motion of the hips, knees, and back was present with no contractures. The patient reproduced the snapping with hip flexion of approximately $90^{\circ}$ while she was in the standing position. The snapping was palpable at the ischial tuberosity and was audible. Pelvic radiographs showed no abnormalities. Magnetic resonance imaging (MRI) demonstrated a partial tear of the proximal hamstring origin with surrounding edema (Fig. 1). While the patient performed the provocative maneuver, dynamic ultrasound with the probe on the ischial tuberosity demonstrated subluxation of a portion of the proximal hamstring origin over the tuberosity, confirming the diagnosis of coxa saltans caused by subluxation of the proximal hamstring origin over the ischial tuberosity (Fig. 2).

The patient underwent physical therapy for hamstring stretching and strengthening for six weeks, but the symptoms persisted. A cortisone injection was then administered over the area of maximal tenderness. The symptoms remained unchanged for several weeks after the injection. A second six-week course of physical therapy was prescribed, but, once again, the symptoms failed to improve. Without resolution of the painful snapping, the patient no longer desired to continue conservative treatment; a limited tenotomy of the lateral portion of the proximal hamstring origin was performed.

The patient was placed in the prone position, with the entire left lower extremity draped free and the surgical table flexed at the hip to allow reproduction of the provocative position. The limited tenotomy was performed through a transverse incision in the gluteal fold centered over the ischial tuberosity ${ }^{3}$. The gluteal fascia was incised transversely, and the gluteus maximus muscle was split bluntly. The sciatic nerve was identified and was protected with a retractor throughout the procedure. The hamstring fascial sheath was identified and split longitudinally; an elongated partial tear, comprising $25 \%$ of the semimembranosus muscle origin, was noted. Snapping of this elongated tendon tear over the ischial tuberosity was recreated intraoperatively with hip flexion (Fig. 3). A tenotomy was performed on the elongated, partially torn tendon. This tendon was released directly from the ischial

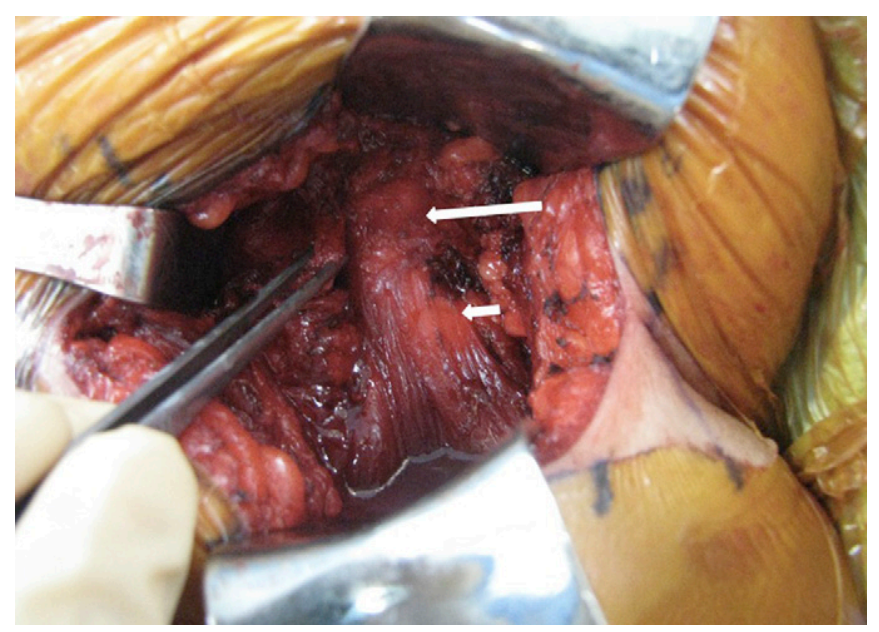

Fig. 3

Forceps localizing the subluxating portion of the hamstrings at the ischial tuberosity with the patient in the prone position. The left lower extremity has been draped free to allow reproduction of the provocative position. The long arrow points to the ischial tuberosity, and the short arrow points to the intact proximal origin of the hamstrings medial to the torn hamstring tendon. 
The Journal of Bone \& Joint Surgery - Jbjs.org Volume 93-A · Number 21 - November 2, 2011
Snapping of the Proximal Hamstring Origin:

A Rare Cause of Coxa Saltans tuberosity, and the remainder of the origin of the hamstrings was spared.

Postoperatively, the patient was permitted weight-bearing as tolerated. Passive and active hip motion was started immediately after surgery without any limitation. Strengthening was started three weeks postoperatively. The patient was free of painful snapping after the tenotomy. She returned to athletic activity three months postoperatively without appreciable hamstring pain or weakness. Nine months after surgery, the patient remained pain-free and no longer experienced hip snapping; she played tennis without limitation.

\section{Discussion}

$\mathrm{W}$ hile the "snapping hip" is a commonly recognized entity, rarely is subluxation of the proximal origin of the hamstrings over the ischial tuberosity considered in the differential diagnosis. A snapping hip is suspected to be due to subluxation of the proximal hamstring origin over the ischial tuberosity when there is a history of audible snapping and pain in the area of the buttock rather than in the anterior aspect of the groin or the lateral portion of the hip ${ }^{2}$. During physical examination, the subluxation of the tendon can be palpated as the patient fully flexes the abducted hip in the standing position.

The hamstring tendons originate on the ischial tuberosity with a common tendon for the biceps femoris and semitendinosus muscles. This common tendon has an oval-shaped insertion, averaging $2.7 \times 1.8 \mathrm{~cm}^{3}$, medial to the semimembranosus muscle. The insertion of the semimembranosus muscle is lateral to the common origin of the biceps femoris and semitendinosus muscles. It is crescent-shaped and has an average size of $2.1 \times 1.1 \mathrm{~cm}^{3}$. During the operative treatment of our patient, the pathology was located on the lateral portion of the origin, and the fibers were contributions from the semimembranosus muscle. However, at this level, the subluxating fibers could not be tracked definitively to a single muscle belly.

Radiographs did not show any pathology in our patient. MRI demonstrated nonspecific changes at the ischial tuberosity consistent with tendinopathy and a partial tear of the lateral portion of the hamstring origin. The utility of MRI is restricted with this diagnosis because of limitations with patient positioning in the MRI scanner. The provocative position is not feasible within the MRI machine, nor is dynamic imaging available with this modality. We believe that dynamic ultrasound is the optimal study for the "snapping hip" caused by subluxation of the proximal hamstring origin at the ischial tuberosity.

To our knowledge, there are no data on the treatment of the "snapping hip" due to subluxation of the proximal ham- string origin except in one case report ${ }^{1}$. Conservative treatment of this condition is the same as the treatment of a high hamstring strain, and includes rest, ice, nonsteroidal antiinflammatory drugs (NSAIDs), and physical therapy ${ }^{4}$. If pain and snapping resolve with these conservative methods, a progressive return to sports with adjuvant low-intensity jogging and cycling can be considered. Cohen and Bradley stated that athletes are able to return to sports after a single hamstring tendon avulsion with retraction up to $2 \mathrm{~cm}^{5}$.

The indications for operative treatment of this condition are not established. Our rationale for the operative procedure that we performed was based on the integrity of the tendon origin on the ischial tuberosity. While clear indications for repair of tears of the hamstring origin are debatable, we utilized those set forth by Cohen and Bradley ${ }^{5}$, in which repair is indicated for a proximal hamstring avulsion involving two or more tendons with $>2 \mathrm{~cm}$ of retraction in a patient who is less than fifty years of age and who desires to continue athletic participation. Based on the nature of this condition, we suspect that it would be improbable to have a completely torn and retracted tendon and, therefore, repair is unlikely to be indicated. It is more plausible that the "snapping hip" resulting from subluxation of the proximal hamstring origin would be caused by tendinopathy or an elongated or partially torn tendon, similar to what was noted in our patient.

Surgical options include partial tenotomy or partial tenotomy with transfer. Athletes with proximal hamstring tendinopathy have been treated with tenotomy of the semimembranosus muscle with suture repair to the biceps femoris muscle with good functional outcomes ${ }^{6,7}$. We did not choose this procedure because our patient was fifty-five years old, was not a high-level athlete, and had only a small tear of the lateral portion of the hamstring origin that was responsible for the painful snapping. As demonstrated in this case report, partial release of the subluxating hamstring tendon from the ischial tuberosity resulted in resolution of the pain as well as the snapping.

Anthony Scillia, MD

Andrew Choo, MD

Edward Milman, MD

Vincent McInerney, MD

Anthony Festa, MD

Office of Research,

St. Joseph's Regional Medical Center,

703 Main Street, Paterson, NJ 07503.

E-mail address for A. Festa: anthonyfestamd@gmail.com

\section{References}

1. Rask MR. "Snapping bottom": subluxation of the tendon of the long head of the biceps femoris muscle. Muscle Nerve. 1980;3:250-1.

2. Allen WC, Cope R. Coxa saltans: the snapping hip revisited. J Am Acad Orthop Surg. 1995;3:303-8.

3. Miller SL, Webb GR. The proximal origin of the hamstrings and surrounding anatomy encountered during repair. Surgical technique. J Bone Joint Surg Am. 2008;90 Suppl 2 Pt 1:108-16.

4. Clanton TO, Coupe KJ. Hamstring strains in athletes: diagnosis and treatment. J Am Acad Orthop Surg. 1998;6:237-48.
5. Cohen S, Bradley J. Acute proximal hamstring rupture. J Am Acad Orthop Surg. 2007;15:350-5.

6. Lempainen L, Sarimo J, Mattila K, Orava S. Proximal hamstring tendinopathyoverview of the problem with emphasis on the surgical treatment. Oper Tech Sports Med. 2009;17:225-8.

7. Lempainen L, Sarimo J, Mattila K, Vaittinen S, Orava S. Proximal hamstring tendinopathy: results of surgical management and histopathologic findings. Am J Sports Med. 2009;37:727-34. 